\title{
Feasibility and Usability of Wearable Devices for Ambulatory Monitoring of the Rehabilitation Process of Older Patients after Hip Fracture Surgery
}

\author{
Dieuwke van Dartel ${ }^{1,2} \mathbb{E}^{a}$, Johannes H. Hegeman ${ }^{1,2}$ and Miriam M. R. Vollenbroek-Hutten ${ }^{1,3}$ \\ ${ }^{I}$ Biomedical Signals and Systems Group, University of Twente, Enschede, The Netherlands \\ ${ }^{2}$ Department of Trauma Surgery, Ziekenhuisgroep Twente, Almelo, The Netherlands \\ ${ }^{3}$ ZGT Academy, Ziekenhuisgroep Twente, Almelo, The Netherlands
}

Keywords: $\quad$ Wearable Devices, Ambulatory Monitoring, Fitbit, MOX, Feasibility, Usability, Older Patients, Hip Fracture.

Abstract: Objective: To assess the feasibility and usability of wearable devices for ambulatory monitoring of older patients during geriatric rehabilitation after hip fracture surgery.

Methods: Patients ( $\geq 70$ years) who were surgically treated for a hip fracture wore the Fitbit Charge 2/HR and the MOX device. Feasibility was assessed by investigating whether real world data gathering revealed sufficient high-quality data. Usability was assessed by 1) evaluating whether changes in the device parameters correlated with changes in clinimetric tests and 2) determining whether the wearable devices properly measured activity.

Results: Data from 67 patients was used to assess feasibility; all patients wore the Fitbit and 33 the MOX. The mean amount of high-quality data was $88.1 \%$ for the Fitbit and $93.6 \%$ for the MOX. Data from 42 patients was used to assess usability; all patients wore the Fitbit and 14 the MOX. A positive progression in clinimetric tests was correlated with an increase in activity parameters. However, the Fitbit often miscalculated the number of steps and the MOX algorithm often misclassified slow walking as standing.

Conclusions: Ambulatory monitoring using the Fitbit and MOX is feasible in older patients with a hip fracture. Concerning the usability, the Fitbit often miscalculated the number of steps. The MOX was more adequate but the activity classification algorithm often misclassified slow walking based on which it is recommended to use the raw data instead.

\section{INTRODUCTION}

The ultimate goal of hip fracture treatment in older patients is functional recovery, which is defined as the patient regaining the premorbid level of functioning (Ceder, 2005; Folbert et al., 2011; Zuckerman, 1996). To achieve this, adequate post-operative rehabilitation during and after the patient's hospital stay is essential (Prestmo et al., 2015). Clinimetric tests are often used to obtain insight into the patient's progress during rehabilitation. These tests assess the patient's physical function, mobility, and cognitive impairment. Scientific studies have used clinimetric tests to provide insight into the rehabilitation process and identify predictive factors for a positive outcome. However, although clinimetrics provide helpful information, they are also static and administered infrequently. Furthermore, it is not always possible to perform a clinimetric test, as patients need a certain level of mobility (Benzinger et al., 2014; Hershkovitz, Beloosesky, \& Brill, 2012; Nygard, Matre, \& Fevang, 2016). As a result, important information about patient recovery during rehabilitation might be missed, with the consequence that treatment is not adjusted at the right time and recovery is suboptimal. Therefore, there is a need for a better, continuous, and accurate way to monitor older hip fracture patients during rehabilitation.

One possible solution is the use of wearable devices. Wearable devices are small, portable, bodyfixed sensors that can be used for continuous ambulatory monitoring of bodily signals. In the case of hip fracture rehabilitation, most ambulatory

https://orcid.org/0000-0002-3556-4522 
monitoring is performed in the physical activity domain. Some scientific studies of the rehabilitation process in older hip fracture patients have already investigated the added value of physical activity monitoring. However, these studies monitored patients for only a few days during hospital stay or rehabilitation instead of continuously throughout the whole rehabilitation process, which lasts for several weeks (Bakker, Blokhuis, Meeks, Hermens, \& Holtslag, 2014; Benzinger et al., 2014; Davenport et al., 2015; Fleig et al., 2016; Keppler et al., 2020; Schmal et al., 2018; Talkowski, Lenze, Munin, Harrison, \& Brach, 2009; Taylor, Peiris, Kennedy, \& Shields, 2016). Other studies measured physical activity in older patients who returned to the community setting, but again only for a few days (Fleig et al., 2016; Resnick et al., 2011; Taraldsen et al., 2015). There is limited information on whether it is feasible or useful to monitor patients throughout the entire rehabilitation period using wearable devices.

Armitage et al. recently assessed the feasibility and acceptability of an activity tracker worn as a pendant for the continuous monitoring of older patients (Armitage et al., 2020). In that study, patients discharged to their home after surgery and patients temporarily discharged for geriatric rehabilitation were continuously monitored for 16 weeks. Results showed that the activity tracker was able to monitor meaningful activity data. However, many patients were unwilling to wear it and, therefore, patient recruitment and retention was low. Therefore, the aim of this study was to assess the feasibility and usability of wrist-worn and thigh-worn wearable devices for the continuous monitoring of older patients during the entire rehabilitation period after hip fracture surgery. Feasibility will be assessed by determining whether real world data gathering revealed sufficient highquality data to monitor a patient's rehabilitation progression. Usability will be assessed by 1) evaluating whether changes measured with wearable devices correlate with changes in the standard clinimetric tests and 2) by determining whether the wearable devices properly assess different activities by comparing recorded data with observations made during therapy sessions.

\section{METHODS}

\subsection{Subjects}

This study included patients aged 70 years or older who received surgery for their hip fracture at the department of Trauma Surgery in Ziekenhuisgroep
Twente (ZGT). Patients with severe cognitive impairment, total hip replacement, a pathological or periprosthetic fracture, terminal illness, or contact isolation were excluded. Where possible, patients were enrolled in the study one day post-surgery; if not possible, inclusion took place one day before the patient was discharged for rehabilitation to one of the three collaborating skilled nursing homes (TriviumMeulenbeltZorg, Carintreggeland, and ZorgAccent). If an included patient was not admitted to one of three collaborating nursing homes, measurements were only taken during the hospital stay. This study was part of the "Up\&Go after a hip fracture" project. All patients gave written informed consent to participate. This study was approved by the ethical review committee of ZGT and the Medical Research Ethics Committee Twente.

\subsection{Continuous Monitoring}

Patients were continuously monitored during their entire hospital stay and/or rehabilitation stay at the nursing home. We initially started monitoring patients with the Fitbit Charge 2 / HR (Fitbit Inc., San Francisco, CA, USA), which are wrist-worn community-based activity trackers that were preferably placed on the patient's non-dominant wrist. The Fitbit contains a 3D-accelerometer and photoplethysmography in order to measure the number of steps a patient takes and the patient's heart rate, respectively. The Fitbit was connected via Bluetooth to the Fitbit App on a mobile phone to access the step count and heart rate data.

After a few months of the study, we began monitoring any newly enrolled patient with a MOX device in addition to a Fitbit, since the Fitbit is not able to monitor time spent in different postures. The MOX (model MMOXX1) is a small, single-unit, dust- and waterproof device $(35 \times 35 \times 10 \mathrm{~mm})$ that continuously monitors physical activity throughout the day (Maastricht Instruments BV, the Netherlands). The MOX contains a 3Daccelerometer, has a sample frequency of $25 \mathrm{~Hz}$, and was attached to the anterior thigh, $10 \mathrm{~cm}$ above the knee of the fractured leg, with a plaster. We used the IDEEQ software provided by Maastricht Instrument BV to download the raw acceleration data from the MOX and convert it into continuous activity data, i.e. the number of active minutes (walking) and the number of sedentary minutes (sitting and lying).

\subsection{Assessment of Feasibility}

Feasibility was assessed by calculating the amount of 
high-quality data that was available when older hip fracture patients were continuously monitored. For each patient, we first calculated the amount of missing data for the MOX and Fitbit during daytime (7.00 am to $10.00 \mathrm{pm}$ ) by calculating the number of missing minutes for each hour. There are no guidelines in the literature for how to handle missing data from these devices, so based on our best judgement, we considered an hour as "missing" if more than 10 minutes of data were missing. When more than three hours were missing on a given day, we considered the day as a missing day. Based on the number of missing days, we then calculated the percentage of available data for each patient and the mean percentage across all patients. The first and last day of the measurement period were excluded for all patients because these days were not full measurement days. Data was analysed with MATLAB R2017b (MathWorks, Natick, MA, USA).

\subsection{Assessment of Usability}

Usability was assessed by determining whether the changes in activity parameters correlated with changes measured in clinimetric tests, which are considered the gold standard for evaluating patient recovery. For this part of the study, we only used data of the patients monitored during rehabilitation at the nursing home. For each patient the number of active minutes, the number of sedentary minutes, and the number of steps per day were calculated. The parameters were then used in linear regression, with time as a dependent variable, to calculate the slope. The slope was used to determine if the patient's progression was positive or negative for each activity parameter. "Positive progression" was defined as cases where the number of active minutes and the number of daily steps have a positive slope and the number of sedentary minutes a negative slope.

Activity progression was compared to results from the following clinimetric tests: Timed Up and Go test (TUG), 10 Meter Walk Test (10MWT), Functional Ambulation Categories (FAC), Katz Index of Independence in Activities of Daily Living (Katz-ADL) and Barthel Index (BI).

The TUG and 10MWT are both functional capacity tests. For the TUG patients were instructed to stand up from a chair, walk three meters, turn around, walk back to the chair, and sit down again. The time (in seconds) that it took to perform the test was used as an outcome measure. For the 10MWT the patient's gait speed $(\mathrm{m} / \mathrm{sec})$ was assessed over a 10 meter distance and used as an outcome measure.

The FAC, Katz-ADL and BI are functional perfor- mance tests. The FAC assessed the patient's ability to walk and ranged from 0 (not functionally able to walk) to 5 (walk independently). The TUG and $10 \mathrm{MWT}$ tests could only be performed with $\mathrm{FAC} \geq 3$. The Katz-ADL and BI assessed the patient's independence in activities of daily living (ADL). The Katz-ADL ranged from 0 (completely independent) to 6 (completely dependent) and the BI from 0 (completely dependent) - 20 (completely independent).

To calculate patient progression for each clinimetric test, we calculated the difference between the test score obtained at discharge from the rehabilitation department and the test score obtained at admission to the rehabilitation department. Differences were expressed as a percentage of the initial (admission) score, resulting in measurements for $\Delta \mathrm{TUG}, \Delta 10 \mathrm{MWT}, \Delta \mathrm{FAC}, \Delta \mathrm{Katz}-\mathrm{ADL}$, and $\Delta \mathrm{BI}$. A patient exhibited a "positive progression" during rehabilitation if $\triangle 10 \mathrm{MWT}, \triangle \mathrm{FAC}$, and $\triangle \mathrm{BI}$ were positive and $\triangle T U G$ and $\triangle$ Katz-ADL were negative. Subsequently, we calculated Pearson's or Spearman's correlation coefficient between the slope of the activity parameters over time and $\Delta \mathrm{TUG}, \Delta 10 \mathrm{MWT}$, $\Delta \mathrm{FAC}, \Delta \mathrm{Katz}-\mathrm{ADL}$, and $\Delta \mathrm{BI}$ to assess how well results from continuous sensors correlated with results from clinimetric tests.

To assess whether the Fitbit and the MOX correctly identified patient activity as "activity" a researcher observed weekly therapy sessions at the rehabilitation department, with 10 patients observed for a total of 37 sessions. The observer noted the start and end time for each activity (sitting, standing, and walking) and manually counted the number of steps when patients were walking. Results from these direct observations were compared with the activity-data logged by the Fitbit and the MOX. Deviations between the observed and the monitored values were expressed as percentages.

\section{RESULTS}

\subsection{Subjects}

A total of 86 patients were enrolled in this study. Of these patients, 19 did not complete the study; reasons for non-completion included problems with synchronizing the Fitbit $(\mathrm{n}=6)$, choosing not to complete the study $(n=3)$, not wearing the Fitbit $(n=2)$, discomfort of the Fitbit $(n=1)$, an allergic reaction to the MOX plaster $(n=1)$, overall decline in health status $(n=1)$, contact isolation $(n=1)$, death 
during rehabilitation $(\mathrm{n}=1)$, or unknown reasons $(\mathrm{n}=3)$.

The sensor data from the remaining 67 patients was used to assess the feasibility of the sensors. All 67 patients wore the Fitbit. The median measurement period was 24 days (min: 2 days, max: 75 days). Because the MOX measurements were added later in the study, only 33 of the 67 patients also wore the MOX. The median measurement period was 6 days (min: 2 days, max: 75 days).

Data from 42 of the 67 patients was used to assess the usability of the wearable sensors. All 42 patients wore the Fitbit, and 14 of these patients also wore the MOX. The median measurement period was 29 days (min: 11 days, max: 71 days) and 27 days (min: 11 days, max 67 days) for the Fitbit and MOX, respectively. The mean age of the 42 patients was 82 \pm 6 years, and $83 \%$ of the patients were female. Prior to the hip fracture, $69 \%$ of the patients lived independent and $57 \%$ of the patients were able to walk independently. The mean age of the ten patients whose therapy sessions were observed by a researcher was $83 \pm 3$ years, and $70 \%$ of the observed patients were female.

\subsection{Feasibility}

The percentage of available data varied among patients, with a maximum of $100 \%$ data availability for both the Fitbit and the MOX and a minimum of $20 \%$ data availability for the Fitbit and $74 \%$ for the MOX (Figure 1). The mean percentage of available data across all patients were $88.1 \%$ and $93.6 \%$ for the Fitbit and MOX, respectively. Data availability was more variable among patients monitored with the Fitbit compared with the MOX (Figure 1).

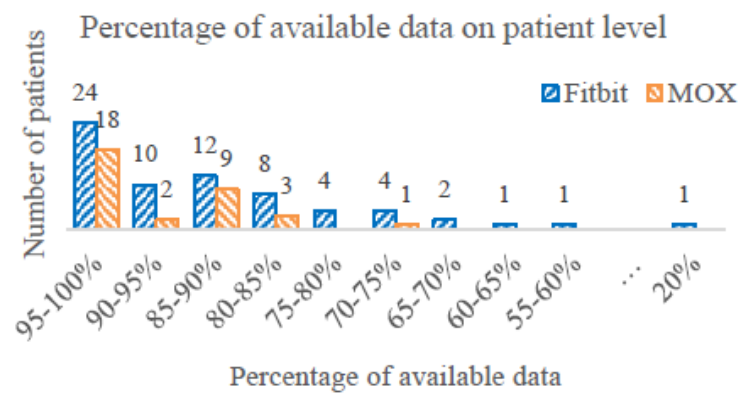

Figure 1: The percentage of available data for each patient for the Fitbit and the MOX.

\subsection{Usability}

Results show that most patients show a positive progression throughout their rehabilitation, measured both with the clinimetric tests as well as with the activity parameters. However, $\triangle 10 \mathrm{MWT}$ is missing for $57 \%$ of the patients and $\Delta \mathrm{TUG}$ for $55 \%$.
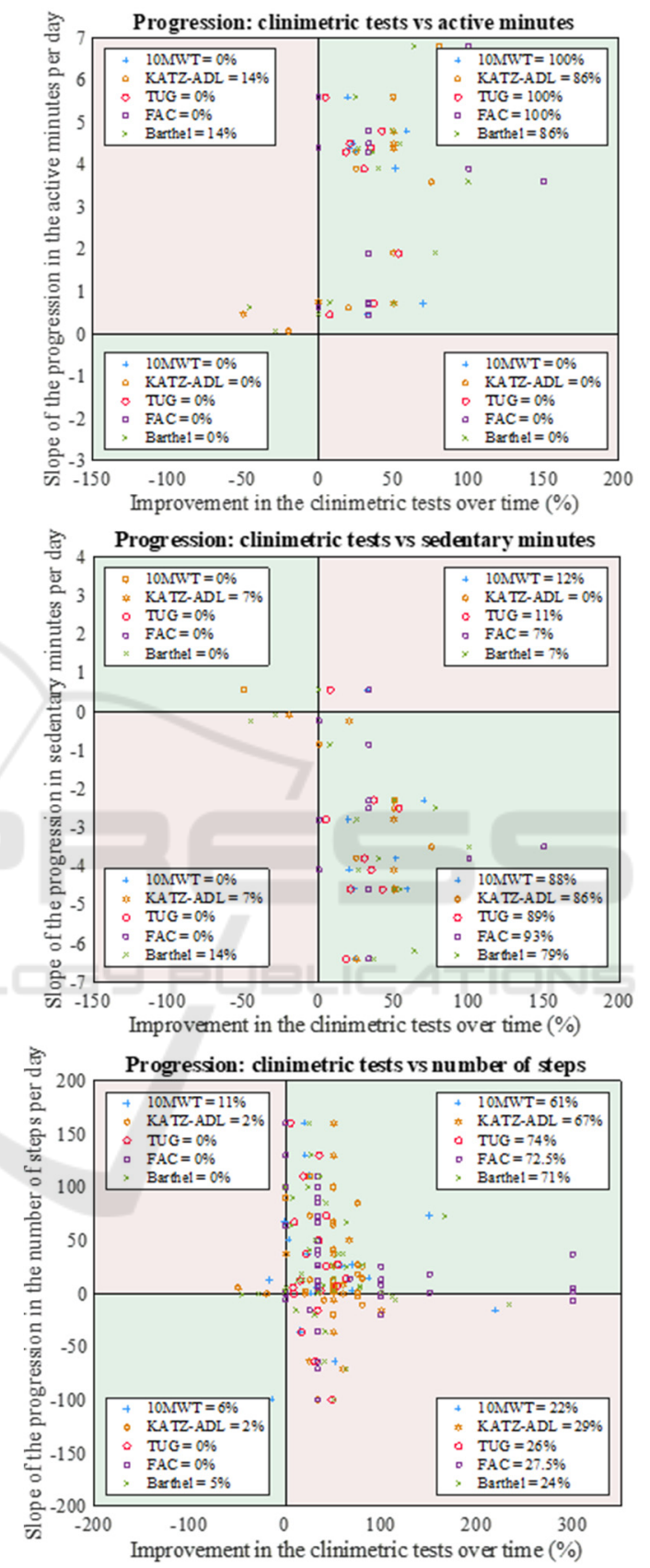

Figure 2: This figure provides a scatterplot for each activity parameter, in which the slope of the activity parameter is compared against the patient's progression in clinimetric tests. The clinimetric data is standardized so that the progression is shown for all the clinimetric tests. The legend in every quarter shows the percentage of patients within that plane. 
Figure 2 presents the scatterplots to compare each patient's progression in the clinimetric tests with their progression in the activity parameters. In each plot, each point represents a patient's result. Different point shapes represent the different clinimetric tests. Each scatterplot is divided into four quarters; patients (points) in the green quarters show the same progression in their activity parameter as in their clinimetric tests. The pink quarters represent those patients with discrepancies between activity parameter and clinimetric tests.

For most patients, the physical activity parameters show the same progression as the clinimetric tests. However, approximately $25 \%$ of the patients show a decrease in the number of steps even though the clinimetric tests indicate a positive progress.

Results from the correlation tests show that for $\triangle$ Katz-ADL there is a moderate negative correlation with the slope of the number of active minutes $(\mathrm{r}=$ $0.66, \mathrm{p}<0.05, \mathrm{n}=14$ ) and a moderate positive correlation with the slope of the number of sedentary minutes $(\mathrm{r}=0.67, \mathrm{p}<0.01, \mathrm{n}=14)$. For $\Delta \mathrm{BI}$ it shows that there is a moderate positive correlation with the slope of the number of active minutes $(r=0.54, p<$ $0.05, n=14)$ and a moderate negative correlation with the slope of the number of sedentary minutes $(\mathrm{r}=$ $0.57, \mathrm{p}<0.05, \mathrm{n}=14)$. No other significant correlations were found between the changes in activity parameter and changes in clinimetric tests (Table 1).

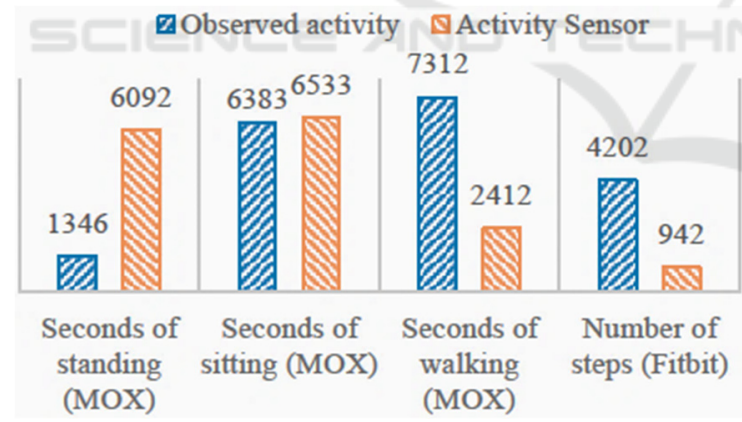

Figure 3: This figure compares the observed activity of patients during their therapy sessions and the activity measured by the Fitbit and MOX. For each measure, the bars show the sum of the activity measure over all observed therapy sessions.

Figure 3 shows how well the activities are measured and recorded by the Fitbit and the MOX compared to direct observations of patient activity. Data from 24 of the 37 observed therapy sessions were used to evaluate the accuracy of the Fitbit's measured number of steps. Not all therapy sessions were used since some sessions did not contain proper step count observations. A total number of 4,202 steps were observed by the researcher across all 24 sessions; however, the Fitbit only counted 942 steps (Figure 3), which means that only $22.4 \%$ of the observed number of steps were correctly measured by the Fitbit. The Fitbit generally counted too few steps when patients were walking with a walker and too many when patients were moving around in a wheelchair.

Data from 33 of the 37 observed therapy sessions were used to compare the activity measured with the MOX to observed activity. During these sessions, the researcher observed a total of 6,383 seconds of sitting, 1,346 seconds of standing, and 7,312 seconds of walking, whereas the MOX measured 6,533, 6,092 , and 2,412 seconds of sitting, standing, and walking, respectively (Figure 3 ). This means that the MOX overestimated the amount of time spent sitting and standing by $2.3 \%$ and $352.6 \%$, respectively, and underestimated the seconds of walking, as only $33 \%$ of the observed second $\mathrm{s}$ of walking were also measured by the MOX.

\section{DISCUSSION}

The aim of this study was to assess the feasibility and usability of the Fitbit Charge and the MOX for continuously monitoring of the physical activity of older patients throughout their rehabilitation period after hip fracture surgery. We found that $78 \%$ of the patients adhere to the sensors and approximately $88 \%$ and $94 \%$ high quality data was available for the Fitbit and MOX measurements, respectively. This suggests that it is feasible to use wearable devices for longterm monitoring and that these devices record enough data to obtain insight in a patient's progression during rehabilitation. We also found that the clinical progression measured using the sensor parameters was generally the same as the progression measured with the standard clinimetric tests, suggesting that the data produced by the Fitbit and MOX is also usable. However, the Fitbit was not always able to properly count the number of steps, especially in patients using a wheelchair or walking aids, and the IDEEQ software for analysing the MOX data often classified slow walking as standing.

\subsection{Feasibility}

Patients in our study were generally open to wearing the Fitbit and the MOX sensors and wore them correctly. Similar results were found by O'Brien et al. who also showed a high acceptability of a wristband activity tracker in older adults (O'brien, Troutman- 
Table 1: Correlation coefficients from the different correlation tests, which tested whether the slope of the activity parameters of the Fitbit and the MOX were correlated with the patient's progression in clinimetric tests. The MOX parameters (slope of the active minutes and slope of the sedentary minutes) were compared to clinimetric tests using Spearman's correlation. The slope of the number of steps was tested using Pearson's correlation with the exception of the comparison with $\Delta$ TUG.

\begin{tabular}{|c|c|c|c|c|c|}
\hline & $\Delta$ 10MWT & $\Delta$ TUG & $\triangle$ FAC & $\Delta$ KATZ-ADL & $\Delta \mathrm{BI}$ \\
\hline \multirow{2}{*}{ Slope number of steps } & $\mathrm{r}=0.02$ & $\mathrm{r}=0.33$ & $\mathrm{r}=-0.31$ & $\mathrm{r}=0.13$ & $\mathrm{r}=-0.14$ \\
\hline & $\mathrm{p}=0.94$ & $\mathrm{p}=0.18$ & $\mathrm{p}=0.06$ & $\mathrm{p}=0.42$ & $\mathrm{p}=0.39$ \\
\hline \multirow{2}{*}{ Slope active minutes } & $\mathrm{r}=-0.2$ & $\mathrm{r}=0.33$ & $\mathrm{r}=0.07$ & $r=-0.66$ & $\mathrm{r}=0.54$ \\
\hline & $\mathrm{p}=0.63$ & $\mathrm{p}=0.38$ & $\mathrm{p}=0.83$ & $\mathrm{p}<0.05$ & $p<0.05$ \\
\hline \multirow{2}{*}{ Slope sedentary minutes } & $\mathrm{r}=0.39$ & $\mathrm{r}=-0.08$ & $\mathrm{r}=-0.28$ & $r=0.67$ & $\mathrm{r}=-\mathbf{0 . 5 7}$ \\
\hline & $\mathrm{p}=0.35$ & $\mathrm{p}=0.83$ & $\mathrm{p}=0.35$ & p $<0.01$ & p $<0.05$ \\
\hline
\end{tabular}

Jordan, Hathaway, Armstrong, \& Moore, 2015). In contrast, Raymond et al. and Armitage et al. found a low acceptability of the sensors used in their study. However, Raymond et al. used an activity tracker that consists of two parts connected via an electrical cable (PAL2) and Armitage et al. used an activity tracker worn in a pendant (Armitage et al., 2020; Raymond, Winter, Jeffs, Soh, \& Holland, 2018). Both devices were no compact sensor, and both have cables, which could explain the discrepancy with our generally high rate of devices acceptance.

We found that wearable devices resulted in more available data than was obtained using clinimetric tests. It is not possible to obtain clinimetric data from every patient since some patients lack the mobility necessary to perform a clinimetric test. This was true in our study, as $\triangle \mathrm{TUG}$ and $\triangle 10 \mathrm{MLT}$ were missing for $55 \%$ and $57 \%$ of the patients, respectively. However, this was not the case for continuous monitoring with the Fitbit and the MOX, for which approximately $88 \%$ and $94 \%$ of the data were available, respectively. This shows the advantage of ambulant sensing as this reveals a high amount of data that do provide a clear insight in the progression of the patient and enables the detection of deterioration at an earlier stage.

Data availabilty was not $100 \%$ in all patients. The main reason for missing data was due to sensor charging; the MOX and Fitbit had a battery life of 7 days and 3-7 days, respectively. The Fitbit exhibited more variability in the amount of available data for each patient, which could also be due to forgetting to synchronize the Fitbit, or due to disturbances in the measurements caused by sweat, movement of the sensor, no proper contact with the skin, or excessive pressure on the skin (Allen, 2007; Jo, Lewis, Directo, Kim, \& Dolezal, 2016).

\subsection{Usability}

Regaining the premorbid level of functioning is the main goal in the rehabilitation of an older hip fracture patient (Ceder, 2005; Zuckerman, 1996), and one way to achieve this is by increasing physical activity.
Correlation tests showed that an improvement on the Katz-ADL and BI tests was correlated with a positive progress in the number of active minutes per day and the number of sedentary minutes per day. This corresponds with previous studies that have shown that physically active patients need less time to regain ADL function, instrumental ADL function, and mobility (Hardy \& Gill, 2005; Talkowski et al., 2009; Willems, Visschedijk, Balen, \& Achterberg, 2017).

The correlations between the activity parameters of the MOX and the other clinimetric tests showed the same directional association, indicating that the general progression recorded by each approach was the same, though these correlations were not significant. This lack of significance can probably be explained by the fact that the $\Delta \mathrm{TUG}$ and $\Delta 10 \mathrm{MWT}$ are focused on the physical capacity of a patient, i.e. what a patient is capable of doing, whereas the MOX is focused on the patient's physical activity, i.e. what a patient actually does. These are two different aspects, and it could occur that a patient is showing less physical activity than he/she is capable of doing, where pain and low motivation are great barriers for being physically active during hip fracture rehabilitation (Benzinger et al., 2014; Resnick et al., 2011; Sims-Gould, Stott-Eveneshen, Fleig, McAllister, \& Ashe, 2017; Talkowski et al., 2009). In addition, the correlation coefficients were assessed on the results of only 14 patients for whom both MOX and clinimetric data was available, which is a very limited sample size.

There were more discrepancies between the progression in the number of steps monitored with the Fitbit and the progression in the clinimetric tests, and none of the correlations were significant. Approximately $25 \%$ of the patients showed a positive progression in their clinimetric tests but a negative progression in their number of steps per day. One potential cause of this discrepancy is that the Fitbit was not able to properly count the number of steps in older patients, as the Fitbit calculated too many steps when a patient was in a wheelchair and too few steps when a patient walked with a walker. This 
miscalculation likely arises because the Fitbit is worn around the wrist. Moving around in a wheelchair results in movement of the wrists, so the Fitbit incorrectly counts this movement as steps. Walking with a walker results in no movement of the wrists, so the Fitbit does not count any steps. Schmal et al. similarly found that step counts were less accurate in patients using mobility aids (Schmal et al., 2018). It is also possible that patients in our study walked too slowly for the Fitbit to accurately count their steps (Schmal et al., 2018); Treacy et al. showed that the Fitbit produced an inaccurate step count relative to the observed step count in a group of slow-walking participants with a mean age of 80 years (Treacy et al., 2017). We therefore suggest using a wearable device located on the lower extremities to monitor the physical activity of older patients.

The MOX is one such device that can be located on the lower extremity. However, the IDEEQ software associated with the MOX device was also unable to properly detect activity in older patients. The algorithm for activity classification by the IDEEQ software was designed based on the activity of two patient populations with a mean age of $54.2 \pm 16.8$ and $60 \pm 9.9$ years old (Annegarn et al., 2011), which is significantly younger than the mean age of the patients monitored in our study ( $\geq 70$ years). This could explain why slow walking was considered as standing, as the threshold for "walking" was set too high to correctly classify it in an older population. However, the IDEEQ software also provides raw acceleration data, which could be used to design casespecific activity classification algorithms.

More broadly, this study showed that continuous monitoring has several advantages over traditional clinimetric tests. First, continuous monitoring is not prone to the ceiling effects common in clinimetric tests. A "ceiling effect" occurs when patients reach a high or maximal score on a clinimetric test at the beginning of rehabilitation, leaving little room for further improvement. A second advantage of continuous monitoring is the lack of "floor effects," which arise when patients are not able to perform a clinimetric test. In this study, we found floor effects for the TUG and the 10MWT, which caused a high percentage of missing data at admission. In contrast, physical activity could be monitored in all patients, despite their level of functioning. Third, continuous monitoring provides more in-depth information about the progression of a patient at all times and is therefore less prone to selectively measuring on a bad or good day.

\section{CONCLUSIONS}

Continuous physical activity monitoring with the use of the Fitbit and the MOX was feasible for older hip fracture patients throughout their rehabilitation program. Older patients were largely willing to wear these devices, resulting in a high amount of available data, and the rehabilitation progression indicated by continuous monitoring of physical activity was similar to the progression measured with clinimetric tests. Continuous monitoring also provided information about the patient's progression, including fluctuations between days and trajectories over time, that could not be obtained from clinimetric tests. However, the Fitbit was less usable than the MOX in a population of older patients. Because the Fitbit is worn around the wrist, it often could not properly measure the number of steps in patients who used mobility aids. The MOX did not have these disadvantages, though we recommend developing a new algorithm that uses the raw accelerometer data to correctly classify the activity of older patients, as the MOX could sometimes identify slow walking as standing. Further research is needed to optimize valid parameter extraction from continuous monitoring devices worn by patients with very low physical activity levels, like those recovering from hip surgery.

\section{ACKNOWLEDGEMENTS}

The Up\&Go after a hip fracture group: M.M.R. Vollenbroek-Hutten, J.H. Hegeman, E.C. Folbert, S. Woudsma, C. de Pagter, M.M. Kemerink op Schiphorst, S. Gommers, T. Oude Weernink, A.J.M. Harperink, A.H.S. Oude Luttikhuis, N. den Braber, C. Pierik, A. Malki, and D. van Dartel.

\section{REFERENCES}

Allen, J. (2007). Photoplethysmography and its application in clinical physiological measurement. Physiological measurement, 28(3), R1.

Annegarn, J., Spruit, M. A., Uszko-Lencer, N. H., Vanbelle, S., Savelberg, H. H., Schols, A. M., Meijer, K. (2011). Objective physical activity assessment in patients with chronic organ failure: a validation study of a new single-unit activity monitor. Archives of physical medicine and rehabilitation, 92(11), 1852-1857. e1851.

Armitage, L. C., Chi, Y., Santos, M., Lawson, B. K., Areia, C., Velardo, C., . . F Farmer, A. J. (2020). Monitoring activity of hip injury patients (MoHIP): A sub-study of the World Hip Trauma Evaluation observational cohort 
study. Pilot and Feasibility Studies, 6(1). doi:10.1186/s40814-020-00612-2

Bakker, A., Blokhuis, T. J., Meeks, M. D., Hermens, H. J., \& Holtslag, H. R. (2014). Dynamic weight loading in older people with hip fracture. Journal of rehabilitation medicine, 46(7), 708-711.

Benzinger, P., Lindemann, U., Becker, C., Aminian, K., Jamour, M., \& Flick, S. (2014). Geriatric rehabilitation after hip fracture. Zeitschrift für Gerontologie und Geriatrie, 47(3), 236-242.

Ceder, L. (2005). Predicting the success of rehabilitation following hip fractures. Disability and rehabilitation, 27(18-19), 1073-1080.

Davenport, S. J., Arnold, M., Hua, C., Schenck, A., Batten, S., \& Taylor, N. F. (2015). Physical activity levels during acute inpatient admission after hip fracture are very low. Physiotherapy Research International, 20(3), 174-181.

Fleig, L., McAllister, M. M., Brasher, P., Cook, W. L., Guy, P., Puyat, J. H., Ashe, M. C. (2016). Sedentary behavior and physical activity patterns in older adults after hip fracture: a call to action. Journal of aging and physical activity, 24(1), 79-84.

Folbert, E., Smit, R., van der Velde, D., Regtuijt, M., Klaren, H., \& Hegeman, J. (2011). Multidisciplinary integrated care pathway for elderly patients with hip fractures: implementation results from Centre for Geriatric Traumatology, Almelo, The Netherlands. Nederlands tijdschrift voor geneeskunde, 155(26), A3197-A3197.

Hardy, S. E., \& Gill, T. M. (2005). Factors associated with recovery of independence among newly disabled older persons. Archives of Internal Medicine, 165(1), 106112.

Hershkovitz, A., Beloosesky, Y., \& Brill, S. (2012). Mobility assessment of hip fracture patients during a post-acute rehabilitation program. Archives of Gerontology and Geriatrics, 55(1), 35-41.

Jo, E., Lewis, K., Directo, D., Kim, M. J., \& Dolezal, B. A. (2016). Validation of biofeedback wearables for photoplethysmographic heart rate tracking. Journal of sports science \& medicine, 15(3), 540.

Keppler, A. M., Holzschuh, J., Pfeufer, D., Neuerburg, C., Kammerlander, C., Böcker, W., \& Fürmetz, J. (2020). Postoperative physical activity in orthogeriatric patients-new insights with continuous monitoring. Injury.

Nygard, H., Matre, K., \& Fevang, J. M. (2016). Evaluation of Timed Up and Go Test as a tool to measure postoperative function and prediction of one year walking ability for patients with hip fracture. Clinical rehabilitation, 30(5), 472-480.

O'brien, T., Troutman-Jordan, M., Hathaway, D., Armstrong, S., \& Moore, M. (2015). Acceptability of wristband activity trackers among community dwelling older adults. Geriatric Nursing, 36(2), S21-S25.

Prestmo, A., Hagen, G., Sletvold, O., Helbostad, J. L., Thingstad, P., Taraldsen, K., . . . Lamb, S. E. (2015). Comprehensive geriatric care for patients with hip fractures: a prospective, randomised, controlled trial. The Lancet, 385(9978), 1623-1633.

Raymond, M. J., Winter, A., Jeffs, K. J., Soh, S.-E., \& Holland, A. E. (2018). Acceptability of physical activity monitoring in older adults undergoing inpatient rehabilitation. Aging clinical and experimental research, 30(8), 1005-1010.

Resnick, B., Galik, E., Boltz, M., Hawkes, W., Shardell, M., Orwig, D., \& Magaziner, J. (2011). Physical activity in the post-hip-fracture period. Journal of aging and physical activity, 19(4), 373-387.

Schmal, H., Holsgaard-Larsen, A., Izadpanah, K., Brønd, J. C., Madsen, C. F., \& Lauritsen, J. (2018). Validation of Activity Tracking Procedures in Elderly Patients after Operative Treatment of Proximal Femur Fractures. Rehabilitation research and practice, 2018.

Sims-Gould, J., Stott-Eveneshen, S., Fleig, L., McAllister, M., \& Ashe, M. C. (2017). Patient perspectives on engagement in recovery after hip fracture: a qualitative study. Journal of aging research, 2017.

Talkowski, J. B., Lenze, E. J., Munin, M. C., Harrison, C., \& Brach, J. S. (2009). Patient participation and physical activity during rehabilitation and future functional outcomes in patients after hip fracture. Archives of physical medicine and rehabilitation, 90(4), 618-622.

Taraldsen, K., Thingstad, P., Sletvold, O., Saltvedt, I., Lydersen, S., Granat, M. H., . . Helbostad, J. L. (2015). The long-term effect of being treated in a geriatric ward compared to an orthopaedic ward on six measures of free-living physical behavior 4 and 12 months after a hip fracture-a randomised controlled trial. $B M C$ geriatrics, 15(1), 160.

Taylor, N. F., Peiris, C. L., Kennedy, G., \& Shields, N. (2016). Walking tolerance of patients recovering from hip fracture: a phase I trial. Disability and rehabilitation, 38(19), 1900-1908.

Treacy, D., Hassett, L., Schurr, K., Chagpar, S., Paul, S. S., \& Sherrington, C. (2017). Validity of different activity monitors to count steps in an inpatient rehabilitation setting. Physical therapy, 97(5), 581-588.

Willems, E., Visschedijk, J., Balen, R., \& Achterberg, W. (2017). Physical Activity, Physical Function and Fear of Falling After Hip Fracture. J Orthop Res Physiother, 3,031 .

Zuckerman, J. D. (1996). Hip fracture. New England journal of medicine, 334(23), 1519-1525. 